University of Wollongong

Research Online

Faculty of Engineering and Information

Faculty of Engineering and Information

Sciences - Papers: Part A

Sciences

$1-1-2013$

Time-domain spectroscopy of novel nematic liquid crystals in the terahertz range

Christian Weickhmann

Darmstadt University of Technology

R Jakoby

Darmstadt University of Technology

Evan Constable

University of Wollongong, evanc@uow.edu.au

R A. Lewis

University of Wollongong, roger@uow.edu.au

Follow this and additional works at: https://ro.uow.edu.au/eispapers

Part of the Engineering Commons, and the Science and Technology Studies Commons

Research Online is the open access institutional repository for the University of Wollongong. For further information contact the UOW Library: research-pubs@uow.edu.au 


\title{
Time-domain spectroscopy of novel nematic liquid crystals in the terahertz range
}

\begin{abstract}
The dielectric properties of nematic liquid crystal (LC) mixtures are measured from $300 \mathrm{GHz}$ to $1500 \mathrm{GHz}$. Measurements are performed in a standard THz TDS setup. Refractive index and extinction coefficient for parallel and perpendicular orientation are calculated and compared to results obtained at $19 \mathrm{GHz}$. The investigated mixtures are of interest for Terahertz devices as their properties stay almost constant from $19 \mathrm{GHz}$ to $1500 \mathrm{GHz}$.
\end{abstract}

\section{Keywords}

domain, time, spectroscopy, novel, nematic, liquid, crystals, terahertz, range

Disciplines

Engineering | Science and Technology Studies

\section{Publication Details}

Weickhmann, C., Jakoby, R., Constable, E. \& Lewis, R. A. (2013). Time-domain spectroscopy of novel nematic liquid crystals in the terahertz range. International Conference on Infrared, Millimeter, and Terahertz Waves, IRMMW-THz (pp. 1-2). IEEE. 


\title{
Time-Domain Spectroscopy of Novel Nematic Liquid Crystals in the Terahertz Range
}

\author{
Christian Weickhmann*, Rolf Jakoby*, Evan Constable ${ }^{\dagger}$ and R. A. Lewis ${ }^{\dagger}$ \\ *Technische Universität Darmstadt, Institute of Microwave Engineering and Photonics, Merckstr. 25, 64283 Darmstadt, Germany \\ Email: weickhmann@imp.tu-darmstadt.de \\ ${ }^{\dagger}$ University of Wollongong, Institute for Superconducting and Electronic Materials, Wollongong NSW 2522, Australia
}

\begin{abstract}
The dielectric properties of nematic liquid crystal (LC) mixtures are measured from $300 \mathrm{GHz}$ to $1500 \mathrm{GHz}$. Measurements are performed in a standard THz TDS setup. Refractive index and extinction coefficient for parallel and perpendicular orientation are calculated and compared to results obtained at $19 \mathrm{GHz}$. The investigated mixtures are of interest for Terahertz devices as their properties stay almost constant from $19 \mathrm{GHz}$ to $1500 \mathrm{GHz}$.
\end{abstract}

\section{INTRODUCTION}

Demand for mm-wave and Terahertz devices has been growing in recent years. The need for low-loss, tunable materials is becoming more obvious. Liquid crystals (LC) are organic compounds exhibiting one or more characteristic phases. LCs in their nematic phase have been used for microwave applications over the past decade. Their properties can be described using a dielectric tensor $\tilde{n}=\operatorname{diag}\left\{n_{\|}, n_{\perp}, n_{\perp}\right\}$ with respect to the mean spatial orientation of an ensemble of LC molecules (director) [1]. As the director aligns depending on low-frequency magnetic or electric steering fields, effective refractive index for a given $\mathrm{THz}$ field orientation can be tuned between the two extreme states $n_{\|}$and $n_{\perp}$.

\section{Measurement Setup}

Characterisation was carried out using a Zomega Z-3 $\mathrm{THz}$ Time Domain Spectrometer. As a reference, a $(2.00 \pm 0.03) \mathrm{mm}$ thick sample of TPX $\AA$ (PMP) is characterised. It shows a refractive index of $n_{\mathrm{TPX}} \approx 1.44$ which is in good agreement with experimental results of $n \approx 1.46$ [2] (see fig. 1).

For this work, four LC mixtures from Merck KGaA (Darmstadt, Germany) were studied: a commercial (GT3-23001) and three non-commercial mixtures. Microwave measurements at $19 \mathrm{GHz}$ yield values shown in table I.

The samples were characterised in a sample holder made of TPX with optically thick windows and sample chamber. Magnets were used in order to align the LC properly. The magnetic $B$ field within the fixture is of the order of $>0.3 \mathrm{~T}$. The inhomogenety of the field is considered negligible.

\section{RESULTS}

We observe very similar results for the refractive indices of all mixtures and find good agreement with the microwave results. For the parallel state, $n_{\|} \approx 1.78$ and $\kappa_{\|}$drops below

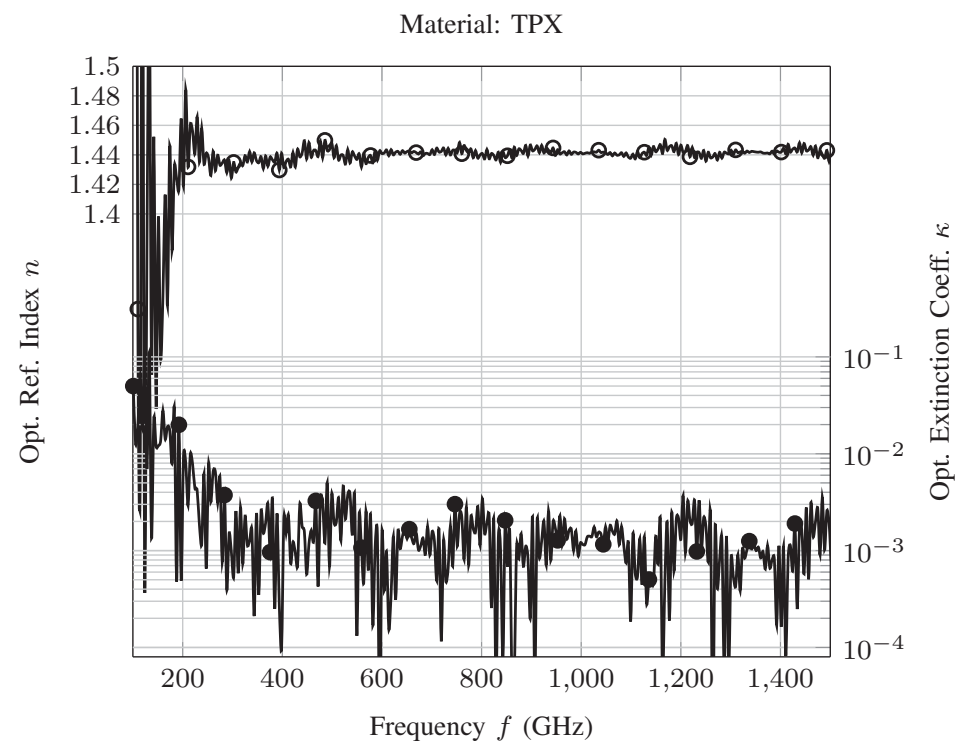

Figure 1. Refractice index $n$ and optical extinction coefficient $\kappa$ of TPX $\left(\multimap n_{\mathrm{TPX}},-\kappa_{\mathrm{TPX}}\right)$ measured as a reference. The result is in very good agreement with literature data

$5 \times 10^{-3}$ from around $400 \mathrm{GHz}$. Furthermore $n_{\perp} \approx 1.55$ while $\kappa_{\perp}$ stays below $1 \times 10^{-2}$. Uncertainties mainly stem from determining the sample thickness $(\Delta d / d \approx \pm 2 \%)$. A noteable deviation from this trend is only visible for the parallel orientation of TUD-325 where most probably an error in the fitting algorithm caused an offset that leads to large systematic error for low frequencies. This needs to be investigated and measurements may need to be repeated. The authors believe that this result is unphysical as there is no indication of a dielectric resonance that could explain this behaviour.

The tuneability (i.e. normalised anisotropy) of all four mixtures, defined as

$$
\tau=\frac{\left|n_{\max }-n_{\min }\right|}{n_{\max }},
$$

is between 14 and $16 \%$.

Below $300 \mathrm{GHz}$ results for $\kappa_{\|, \perp}$ cannot be observed due to a sharp roll-off in signal level when approaching low frequencies and thus a rising noise floor. The trend of almost constant refractive index above $10 \mathrm{GHz}$ is in good agreement with 

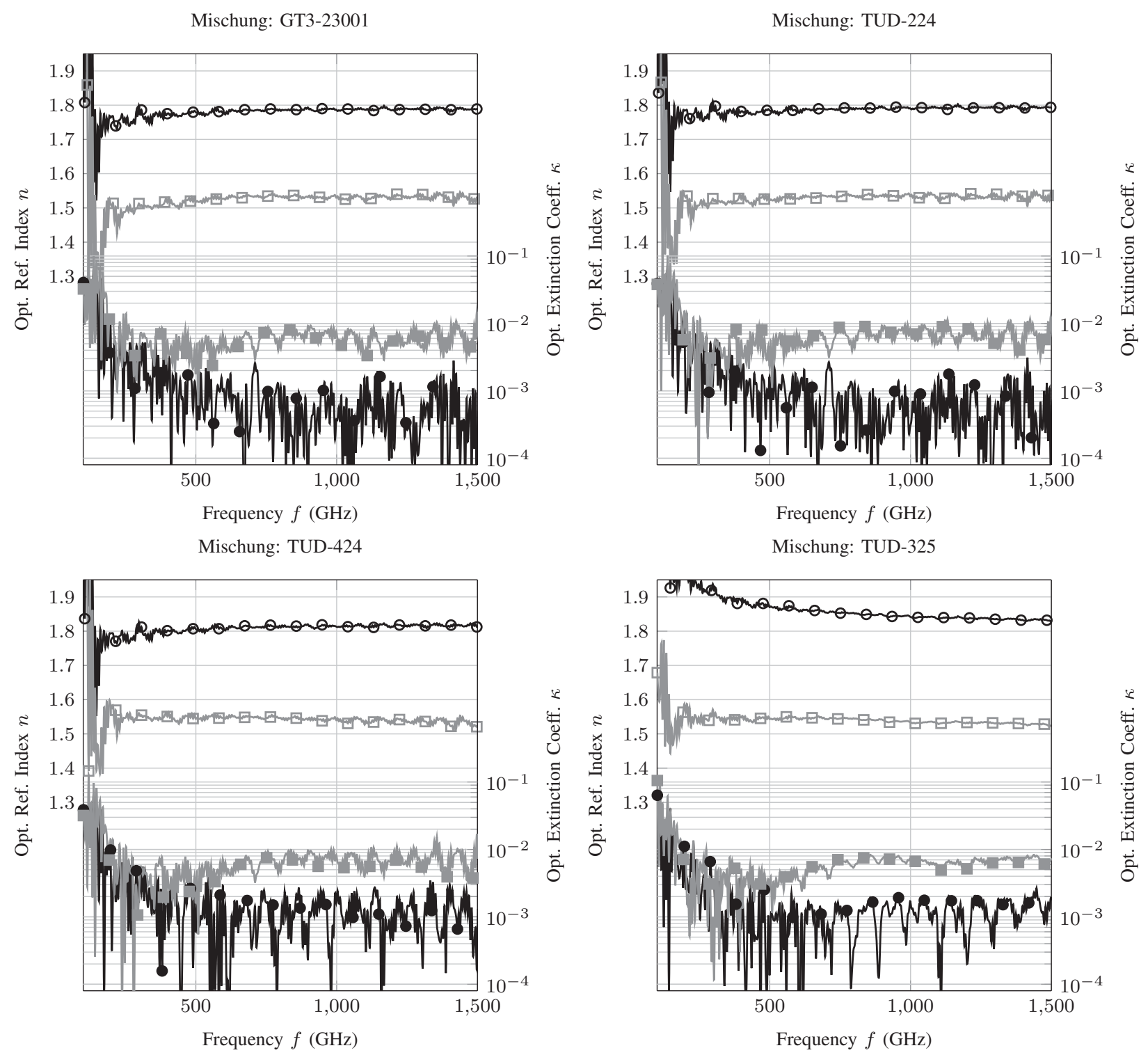

Figure 2. Resulting refractive indices $n$ and extinction coefficients $\kappa$. Refractive index of the parallel state $n_{\|}(-\odot)$ and of the perpendicular state $n_{\perp}$ $(\square-)$, extinction coefficient $\kappa_{\|}(-\bullet)$ and $\kappa_{\perp}(--)$ respectively. The increasing refractive index for TUD-325 for its parallel orientation is most probably due to a numerical issue with the fitting algorithm that needs to be investigated.

Table I

DIELECTRIC PARAMETERS OF EMPLOYED LC MIXTURES (TAKEN AT R.T. AND $f=19 \mathrm{GHz}$, ALL DATA COURTESY MERCK KGAA)

\begin{tabular}{llccc}
\hline Mixture & $\varepsilon_{\|}$ & $\tan \boldsymbol{\delta}_{\|}$ & $\varepsilon_{\perp}$ & $\tan \boldsymbol{\delta}_{\perp}$ \\
\hline GT3-23001 & 3.19 & $3.5 \times 10^{-3}$ & 2.41 & $14.3 \times 10^{-3}$ \\
TUD-224 & 3.18 & $3.0 \times 10^{-3}$ & 2.41 & $12.5 \times 10^{-3}$ \\
TUD-424 & 3.27 & $3.3 \times 10^{-3}$ & 2.45 & $12.6 \times 10^{-3}$ \\
TUD-325 & 3.22 & $2.5 \times 10^{-3}$ & 2.42 & $11.1 \times 10^{-3}$ \\
\hline
\end{tabular}

experience at TU Darmstadt.

\section{CONCLUSiON}

Measurement results of four different liquid crystal (LC) mixtures have been shown to have similar optical refractive indices, high anisotropy (of more than 14\%) and low losses over a very broad range of more than $1 \mathrm{THz}$. The considered materials may therefore be well suited for low loss mm-wave and $\mathrm{THz}$ devices in the future.

\section{REFERENCES}

[1] P.-G. de Gennes and J. Prost, The Physics of Liquid Crystals, 2nd ed. Oxford University Press, 1995.

[2] Tydex J.S.Co., "THz Materials." [Online]. Available: http://www. tydexoptics.com/pdf/THz_Materials.pdf 